\title{
The preparation and clinical application of diagnostic DNA microarray for the detection of pathogens in intracranial bacterial and fungal infections
}

\author{
JINGRONG CAO $^{1}$, SHICHAO GAO ${ }^{1}$, JING CHEN $^{2},{\text { BIN } \text { ZHU }^{3} \text {, RONG MIN }}^{1}$ and PEICHANG WANG ${ }^{1}$ \\ ${ }^{1}$ Department of Clinical Laboratory, Xuanwu Hospital of Capital Medical University, Beijing 100053; \\ ${ }^{2}$ Department of Microbiology, Health Occupation College of Jiangxi, Nanchang, Jiangxi 330201; \\ ${ }^{3}$ Department of Technical Quality, Shanghai BaiO Technology Co., Ltd., Shanghai 200233, P.R. China
}

Received December 16, 2017; Accepted May 11, 2018

DOI: $10.3892 /$ etm.2018.6312

\begin{abstract}
The present study prepared 2 types of DNA diagnostic chips based on 16S ribosomal DNA (rDNA) and 18S-28S rDNA, and evaluated their values in the detection of pathogens in intracranial bacterial/fungal infections. A total of 14 probes of bacteria (Klebsiella pneumonia, Acinetobacter baumannii, Pseudomonas aeruginosa, Escherichia coli, Haemophilus influenza, Stenotrophomonas maltophilia, Neisseria meningitidis, Enterobacter spp., Enterococcus faecalis, Enterococcus faecium, Listeria monocytogenes, Staphylococcus aureus, Streptococcus pneumonia and coagulase negative staphylococcus) and 4 probes of fungi (Candida albicans, Candida tropicalis, Candida glabrata and Cryptococcus neoformans), determined frequently in cerebrospinal fluid (CSF), were designed and used for preparation of microarrays. CSF samples from 88 patients with clinically suspected intracranial infection and standard strains were used to evaluate the chips. The same samples were also analyzed by culture and sequencing. The results demonstrated that the sensitivity, specificity and false-positive rate of the microarray assay compared with culture method were 100 vs. $68.3 \%$ $(\mathrm{P}<0.05), 97.1$ vs. $100 \%$, and 2.9 vs. $0 \%$, respectively. The minimum concentration of detection with the chips was $10 \mathrm{cfu}$ $\mathrm{ml}^{-1}$ for bacteria and $100 \mathrm{cfu} \mathrm{ml}^{-1}$ for fungi. The specificity of the probes was confirmed, and no cross-reaction was detected in the present study. Furthermore, 13 cases were positive in the microarray and negative in culture. However, 4 cases were not identified as clear pathogens and only positive in the $16 \mathrm{~S}$ probe sites. The diagnostic DNA microarray for intracranial
\end{abstract}

Correspondence to: Dr Peichang Wang, Department of Clinical Laboratory, Xuanwu Hospital of Capital Medical University, 45 Changchun Street, Xicheng, Beijing 100053, P.R. China E-mail:pcw1905@126.com

Key words: intracranial infection, pathogens, cerebrospinal fluid, identification, diagnostic DNA microarray infections has proven to be more rapid and sensitive, and it may be a better option for clinical application than culture methods.

\section{Introduction}

Central nervous system (CNS) infections can occur at any age and may be caused by a variety of pathogens, of which bacteria and fungi are the most prevalent $(1,2)$. With rising antibiotic resistance and various surgical operations, pathogens are adapting accordingly (2-4). Previous studies (5-7) have demonstrated that 8-10\% of the regional population (including Egypt, Iran and China) has experienced bacterial and fungal infections of CNS, which expose patients to a higher risk of disability and mortality. Therefore, an accurate, more rapid etiologic identification is key to diagnosis and treatment. However, previous studies $(1,2,5,6,8)$ have reported that the majority of intracranial infections were not clearly diagnosed early enough during hospitalization, which may partially be attributed to limits of traditional detection methods. The conventional methods of cerebrospinal fluid (CSF) examination, including culture and microscopy, have a limited role in severe infections due to its low positive rate $(8-20 \%$ in the majority of studies) and time-consuming process (2-7 days) (6,7,9-11). A number of novel etiological methods have been proposed as a supplement or substitute for the conventional technique, such as polymerase chain reaction (PCR), quantitative PCR $(5,11)$, multiplex PCR techniques $(3,12)$ and DNA sequencing based on $16 \mathrm{~S}$ ribosomal DNA (16S rDNA) in bacteria or the internal transcribed spacer (ITS) in fungi $(6,13-16)$. To the best of our knowledge, PCR and multiplex PCR may only be effective for detecting common clinically suspected organisms $(3,15)$. Furthermore, using more primers will decrease the sensitivity and specificity (15). Finally, DNA sequencing of CSF in clinical application has been limited because of the high cost and low specificity associated with second-generation sequencing $(15,17)$.

Thus, a more rapid, broad-spectrum, accurate, and relatively inexpensive screening method that identifies pathogens from CSF is required. Previous studies $(18,19)$ have demonstrated the diagnostic value and importance of DNA microarray in 
the identification of viral CNS infections. This technology has been proposed to have the potential to simultaneously detect a large number of species with high specificity and has already resulted in its widespread adoption in clinical practice for the diagnosis of viral infections (20-22). However, previous studies have not examined if this technique can simultaneously detect bacterial and fungal CNS infections. The present study established two gene chips, containing 18 probes selected based on specific DNA sequence, to de bacterial and fungal CNS infections and to assess their clinical application value.

\section{Materials and methods}

Clinical CSF samples and strains. A total of 88 CSF samples were collected from patients with suspected CNS infections and analyzed with DNA microarray from January 2014 to July 2016 at Xuanwu Hospital, Capital Medical University (Beijing, China). The present study was approved by the Ethics Committee of Xuanwu Hospital, Capital Medical University. All patients provided written informed consent. The clinical diagnosis of CNS infection was based on the Harrison standard (23), combined with certain risk factors (CSF leak and surgery), clinical manifestations (fever and headache), and CSF examination results (white blood cell count, glucose and protein). The reference strains purchased from American Type Culture Collection (ATCC; Manassas, VA, USA) were as follows: Streptococcus pneumoniae strain ATCC 49619, S. aureus strain ATCC 29213, E. coli strain ATCC 25922, Listeria monocytogenes strain ATCC 7644, Enterococcus faecalis strain ATCC 29212, K. pneumonia strain ATCC 700603, P. aeruginosa strain ATCC 27853, Enterobacter cloacae strain ATCC 700323, Haemophilus influenzae strain ATCC 49766, Cryptococcus neoformans strain ATCC 32269 , Candida albicans strain ATCC 10231, Candida tropicalis strain ATCC 14058 and Candida glabrata strain ATCC 15126. The clinical isolates (Enterococcus faecium, A. baumannii, Stenotrophomonas maltophilia, coagulase negative staphylococcus, Neisseria meningitides, Burkholderia cepacia, Candida parapsilosis, Candida krusei, Cryptococcus gattii) used in the study were collected and identified by Vitek 2 Compact (bioMerieux SA, Marcy-l'Étoile, France), Matrix Assisted Laser Desorption Ionization Time of Flight Mass Spectrometry (MALDI-TOF MS; Bruker Daltonics; Bruker Corporation, Billerica, MA, USA) and DNA sequencing (24).

DNA extraction from strains and CSF. DNA of bacterial and fungal strains were extracted using Bacterial Genomic DNAiso kit (DP302; Tiangen Biotech Co., Ltd., Beijing, China) and Yeast DNAiso kit (DP307; Tiangen Biotech Co., Ltd., Beijing, China) according to the manufacturer's protocol. Each CSF sample (1-2 ml) was centrifuged at $12,000 \mathrm{x} \mathrm{g}$ for $5 \mathrm{~min}$ at room temperature $\left(15-25^{\circ} \mathrm{C}\right)$. The supernatant was discarded and the sediment was pretreated using QIAamp UCP Pathogen Mini kit (Qiagen GmbH, Hilden, Germany), and DNeasy ${ }^{\circledR}$ Blood \& Tissue kit (Qiagen $\mathrm{GmbH}$ ) was used to extract the sample DNA. The DNA was stored at $-20^{\circ} \mathrm{C}$.

Primer design and PCR amplification. Based on a previous study (15), the bacterial and fungal universal primers, $16 \mathrm{~S}$ rRNA and ITS, were designed to be capable of producing
PCR products from standard strains and common organisms causing CNS infection as follows: 27F (forward, 5'-AGAGTT TGATCCTGGCTCAG-3') and 1492R (reverse, 5'-GGYTAC CTTGTTACGACTT-3'), ITS1 (forward, 5'-GCCGTAGGT GAACCTGCGG-3') and ITS4 (reverse, 5'-TCCTCCGCTTAT TGATATGC-3') were used for bacteria and fungi amplification, respectively. Other pairs of primers adding biotin were developed for amplification and hybridization as follows: B1 (forward, 5'-CCTACGGGAGGCAGCAG-3') and B2 (reverse, 5'biotin-TACGGYTACCTTGTTACGACTT-3'; where Y is C or T), P1 (forward, 5'-CAATAAGCGGAGGAAAAGAAA C-3') and P2 (reverse, 5' biotin-ACTCCTTGGTCCGTG TTTCA-3') were used for bacteria and fungi amplification, respectively. PCR was performed according to the protocol of a previous study (15) with a slight modification of annealing temperature of $56^{\circ} \mathrm{C}$ for $25 \mathrm{sec}$ and extension of $72^{\circ} \mathrm{C}$ for $25 \mathrm{sec}$. The PCR products were analyzed using $1 \%$ agarose gel electrophoresis with ultraviolet gel imaging system (Syngene, Frederick, MD, USA), and the amplification bands of fungi and bacteria were observed.

Synthesis of probes. The specific DNA sequences were screened for 14 types of bacteria and 4 fungi from GenBank (http://www.ncbi.nlm.nih.gov/genbank/), using the software Primer Premier 5.0 (Premier Biosoft International, Palo Alto, CA, USA) to design probes. The $16 \mathrm{~S}$ universal probes as a positive reference for all bacteria were designed according to the sequences of conserved domain of $16 \mathrm{~S}$ rDNA, and specific primers or probes were designed based on the sequence of variable domain of $16 \mathrm{~S}$ rDNA. With the exceptions of E. coli and K. pneumonia, the genus probe was designed for other enterobacteria due to high homology. Each probe comprised a modified 19-33 amino acid sequence followed by a spacer of (Poly-dt $)_{16}$ or (Poly-da $)_{16}$ and a stretch of specific sequence (Table I). All primers and probes were synthesized by Shanghai Invitrogen Biotechnology; Thermo Fisher Scientific, Inc. (Waltham, MA, USA). The probe sequence was verified through the comparison of the probe sequence with the PCR product sequence in standard strains and clinical isolates.

Microarray preparation and hybridization. Each array contained repeated triplicate identical sets of probes and arranged in a bacteria-specific matrix of $7 \times 12$ or fungi-specific matrix of $3 \times 9$ (Fig. 1). Each matrix had 'landing-light' spots (spots of an internal control probe or a coordinate probe; Shanghai BaiO Technology Co., Ltd., Shanghai, China) made with cyanine 3 (Cy3)- or Cy5-labeled nucleotides to mark the array orientation. Following labeling the product, both control and sample DNAs were processed with a Hybrid chromogenic reagent kit (BST03021; Shanghai BaiO Technology Co., Ltd.) and hybridized using a BaiO ${ }^{\circledR}$ e-Hyb Automatic hybrid instrument (BSE03011; Shanghai BaiO Technology Co., Ltd.) according to the manufacturers' protocol. The hybridization temperatures of bacterial and fungal chips were 42 and $43^{\circ} \mathrm{C}$, respectively, with each occurring for $30 \mathrm{~min}$. Hybridization and color-substrate reaction procedures are listed in Table II.

The hybridized chip was scanned in $\mathrm{BaiO}^{\circledR}$ BE-2.0 Biochip reading meter (BSE01011; Shanghai BaiO Technology Co., 
Table I. Sequences of microarray hybridization probes for bacteria and fungi.

\begin{tabular}{|c|c|c|}
\hline No. of spot & Name & Probe sequence $\left(5^{\prime}-3^{\prime}\right)$ \\
\hline \multicolumn{3}{|l|}{ Bacteria } \\
\hline 1 & Klebsiella pneumonia & NH2- $\mathrm{ttt} t \mathrm{tt} t \mathrm{tt} t \mathrm{tt} t \mathrm{ttAGCTAATACCGCATAATGTCGCAAGACCAAAGT}$ \\
\hline 2 & Acinetobacter baumannii & NH2-tttttttttttttttCGGTCGCAAGACTAAAACTCAA \\
\hline 3 & Pseudomonas aeruginosa & NH2-tttttttttttttttCCAAAAGCTACTGAGCTAGAGTACGGTA \\
\hline 4 & Escherichia coli & NH2-tttttttttttttttCGGTTTGTTAAGTCAGATGTG \\
\hline 5 & Enterobacter spp. & NH2-tttttttttttttttCGGGGAGGAAGGTGTTGTGGTTAAT \\
\hline 6 & Haemophilus influenzae & NH2-tttttttttttttttGATGTGTTAATAGCACATCAAATTGACGTT \\
\hline 7 & Stenotrophomonas maltophilia & NH2- $\mathrm{ttttttttttttttCGCTAATACCGCATACGACCTACGGGTGAAAGC}$ \\
\hline 8 & Neisseria meningitidis & NH2-tttttttttttttttCAACCTGATTGCTTGGTAGCGTAG \\
\hline 9 & Enterococcus faecalis & NH2-tttttttttttttttCGTTAGTAACTGAACGTCCCCTG \\
\hline 10 & Enterococcus faecium & NH2-tttttttttttttttATGCAAGTCGAACGCTTCTTTTTCCACCGG \\
\hline 11 & Listeria monocytogenes & NH2-ttttttttttttttAGAACAAGGATAAGAGTAACTGC \\
\hline 12 & Staphylococcus. aureus & NH2-ttttttttttttttttAGAACATATGTGTAAGTAACTGTGCACATC \\
\hline 13 & Streptococcus. pneumoniae & NH2-tttttttttttttttAGAAGAACGAGTGTGAGAGTGGAAAGTTCAC \\
\hline 14 & Coagulase negative staphylococcus & NH2-tttttttttttttttGATGAAGGTCTTCGGATCGTAAAACTCTGTTAT \\
\hline 15 & Blank control & - \\
\hline 16 & Gram-negative & NH2-ttttttttttttttCTGATGCAGCCGCGTGTGTGAAG \\
\hline 17 & Gram-positive & NH2-ttttttttttttttGATGACGTCAAATCATCATGCCCCTTATG \\
\hline 18 & Bacterial Universal probe & NH2-ttttttttttttttttAACAGGATTAGATACCCTGGTAGTCCA \\
\hline 19 & Negative control & NH2-tttttttttttttttATTTGTCTTTGTAGATCTTCCCT \\
\hline \multicolumn{3}{|l|}{ Fungi } \\
\hline 1 & Candida albicans & NH2-tttttttttttttttGCATGCTGCTCTCTCGGG \\
\hline 2 & Candida tropicalis & NH2-ttttttttttttttACTGGCTCTTTCAGAGTCCGA \\
\hline 3 & Candida glabrata & NH2-tttttttttttttttACTGGTACCTTTGGTGCCCGA \\
\hline 4 & Cryptococcus neoformans & NH2-tttttttttttttttTGACACGATCACCAGTGCTC \\
\hline 5 & Fungal universal probe & NH2-аaаaаaаaаaаaаaаaTGGGTGGTAAATTCCATCTAAAGC \\
\hline 6 & Blank control & - \\
\hline
\end{tabular}

\section{A 000000000}

\section{$B$}

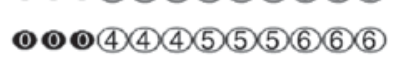

$000(7)(7)(7)(8)(8)(8)(9)(9)(9)$

\section{4}

\section{0}

-00 (10)(10)(11) (11) (11) (12) (12) (12) ○○-(13)(13)(13) (14) (14) (14) (15)(15)(15) ○๐の (16) (16) (16) (17) (17) (17) (18) (18) (18) 0 0 0 (19)(19)(19)(15)(15)(15)(15)(15)(15)

Figure 1. The probe site arrangement of the bacteria and fungi gene-detecting chips. (A) Fungi: 1, Candida albicans; 2, Candida tropicalis; 3, Candida glabrata; 4, Cryptococcus neoformans; 5, ITS; and 6, blank control. (B) Bacteria: 1, Klebsiella pneumoniae; 2, Acinetobacter baumannii; 3, Pseudomonas aeruginosa; 4, Escherichia coli; 5, enterobacter; 6 , Haemophilus influenzae; 7, Stenotrophomonas maltophilia; 8, Neisseria meningitidis; 9, Enterococcus faecalis; 10, Enterococcus faecium; 11, Listeria monocytogenes; 12, Staphylococcus aureus; 13, Streptococcus pneumoniae; 14, Coagulase negative staphylococci; 15, blank control; 16 , Gram-negative bacteria; 17, Gram-negative bacteria; 18, 16S; 19, experimental water; 0 , internal control probes.

Ltd.). Image analysis, fluorescence signal intensity detection and quantification of each probe set was performed with BaiO $^{\circledR}$ Gene chip image analysis software V2.4 (Shanghai BaiO Technology Co., Ltd.). The identification of the infected

specimens was determined, with $\geq 2$ of the bacterial and fungal probes on the array displaying positive hybridization signals.

Analytical sensitivity, specificity and reproducibility of microarray. The analytical sensitivity and specificity of the bacterial and fungal assay was calculated by analysis and comparison with the identified results of culture and sequencing method. A total of 18 clinical isolates (Stenotrophomonas maltophilia, Burkholderia cepacia and Candida parapsilosis; 6 isolates each) with no probes located in the chips, were used to analyze specificity. In addition, the following closely related type and reference strains were analyzed the analytical specificity of the assay: K. pneumoniae and E. coli, P. aeruginosa and Stenotrophomonas maltophilia, etc. Furthermore, genomic DNA of 2 types of pathogens with the sequenced isolates or reference strains were mixed and used as templates for testing the cross-reaction. The detection limit was determined with a 10-fold dilution series, ranging from 1 to 100,000,000 copies of the study strains. To test the reproducibility of the assay, all reference strains and sequenced clinical strains (S. aureus, E. coli and Cryptococcus neoformans) were analyzed and evaluated. DNA extraction, PCR amplification and gene chip hybridization experiment were repeated 6 times, in the same 
Table II. Protocols of bacterial and fungal chip hybridization.

\begin{tabular}{|c|c|c|c|c|c|}
\hline Procedure & Reagent & Volume $(\mu 1)$ & Time (min) & Sampling times & Temperature $\left({ }^{\circ} \mathrm{C}\right)$ \\
\hline 1 & Prehybridization solution & 1,200 & 5 & 1 & 42,43 \\
\hline 2 & Hybridization solution & 200 & 30 & 1 & 42,43 \\
\hline 3 & Wash solution 1 & 800 & 6 & 2 & 42,43 \\
\hline 4 & Wash solution 2 & 1,600 & 5 & 2 & 28 \\
\hline 5 & Antibody solution & 200 & 20 & 1 & 28 \\
\hline 6 & Wash solution2 & / & 5 & 2 & 28 \\
\hline 7 & Wash solution 3 & 400 & 3 & 1 & 28 \\
\hline 8 & Color-substrate solution & 200 & 20 & 1 & 42,43 \\
\hline 9 & Prehybridization solution & / & 2 & 2 & 28 \\
\hline
\end{tabular}

All reagents listed were provided as part of the $\mathrm{BaiO}^{\circledR} \mathrm{e}-\mathrm{Hyb}$ Automatic hybrid instrument kit (Shanghai BaiO Technology Co., Ltd., Shanghai, China). $42,43^{\circ} \mathrm{C}$ represents the hybridization temperatures of bacteria and fungal chips, respectively.

batch and different batches of microarrays. The clinical sensitivity, specificity, false-positive rate and false-negative rates of microarray technique were evaluated with clinical strains from 88 suspected infection CSF samples and 20 negative CSF samples.

Statistical analysis. All data were analyzed using SPSS 19.0 software (IBM Corp., Armonk, NY, USA), and the comparison of ratios expressed via the $\chi^{2}$ test or Fisher's exact test. $\mathrm{P} \leq$ was considered to indicate a statistically significant difference.

\section{Results}

PCR amplification and probe verification. The universal primers of bacteria and fungi were assessed by PCR and sequencing with standard strains and clinical isolates. The PCR products were confirmed to show clear bands of the appropriate size for fungi and bacteria ( 800 and 1,500 bp, respectively) on agarose gel electrophoresis (Fig. 2). No bands were detected in the negative control and blank control (data not shown). The PCR products of the universal primers and DNA template from standard strains or clinical isolates were sequenced. The sequencing results are consistent with the sequences of bacteria or fungi from GenBank, thus confirming the efficiency and accuracy of designed primers. Furthermore, each probe matched that of the PCR products in all standard strains and clinical isolates.

Microarray hybridization. A total of 13 standard strains and 15 clinical isolates were used to assess the chips. Coded DNA samples from species were randomly selected and hybridized to gene chips, with the typical and specific yellow fluorescent signals produced in corresponding microarray spots (Figs. 3 and 4). The results matched $98 \%$ of the results achieved by conventional detection methods except for some subspecies of coagulase negative Staphylococcus. The negative control displayed no signal.

Assessment of microarray hybridization. The results of mixed hybridization revealed that the designed probes are

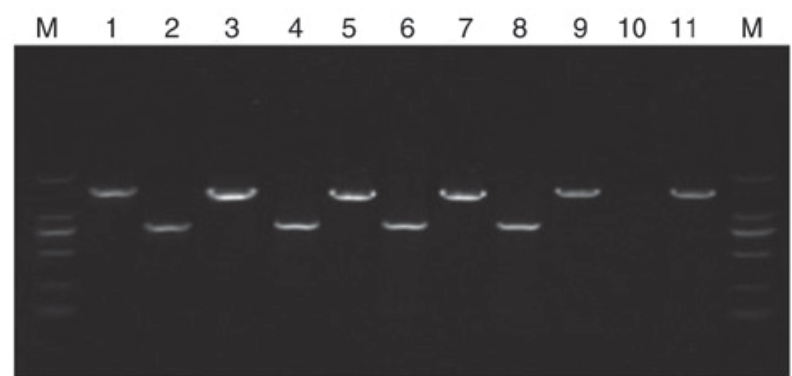

Figure 2. Detection of bacterial and fungal strains using electrophoresis of the polymerase chain reaction products. Lane 1, Escherichia coli; lane 2, Candida albicans; lane 3, Staphylococcus aureus; lane 4, Candida tropicalis; lane 5, Enterococcus faecalis; lane 6, Candida glabrata; lane 7, Klebsiella pneumoniae; lane 8, Cryptococcus neoformans; lane 9, Pseudomonas aeruginosa; lane 10, negative control; lane 11, Enterobacter cloacae; M, DNA marker.

able to detect a mix of 2 types of bacteria or 2 fungal isolates simultaneously (Figs. 3Q and R, and 4F). Using bacterial products in the fungal microarray, no fluorescent signals were detected, and vice versa. No cross-reactions with bacterial species (K. pneumoniae and E. coli) tested and fungal species (Candida albicans and Candida tropicalis) tested by chips were detected. In the fixed condition of PCR, the minimum concentration of detection was $10 \mathrm{cfu} \mathrm{ml}^{-1}$ for Gram-negative bacteria, and $100 \mathrm{cfu} \mathrm{ml}^{-1}$ for Gram-positive bacterial and fungal species. The results also demonstrated that the fluorescent signals were directly associated with the concentration of bacterial and fungal suspension from 10-10,000 $\mathrm{cfu} \mathrm{ml}^{-1}$. However, at the concentration $\geq 100,000 \mathrm{cfu} \mathrm{ml}^{-1}$, all pathogens exhibited the same strength of fluorescent signals. The result of reliability of gene chips with strains demonstrated that all the batch of gene chips reported the same identified results and the coefficient of variation (CV) for within-run and between-run assays were 5.0-8.0 and 5.0-7.0\%, respectively, demonstrating both reproducibility and accuracy.

Primary clinical application of the microarray. A total of 88 CSF samples from patients with suspected intracranial infections and 20 negative CSF samples were selected for microarray 

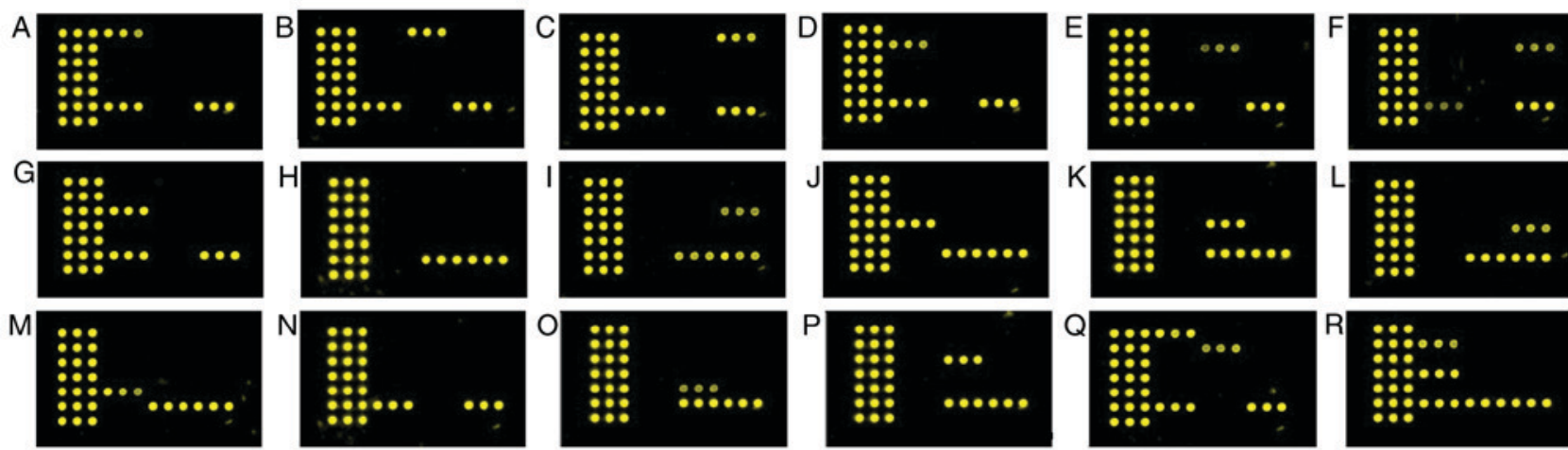

Figure 3. The identification results of bacterial species using DNA microarray. (A) Klebsiella pneumonia; (B) Acinetobacter baumannii; (C) Pseudomonas aeruginosa; (D) Escherichia coli; (E) Enterobacter cloacae; (F) Haemophilus influenzae; (G) Stenotrophomonas maltophilia; (H) gram-positive bacteria; (I) Enterococcus faecalis; (J) Enterococcus faecium; (K) Listeria monocytogenes; (L) Staphylococcus aureus; (M) Streptococcus pneumonia; (N) gram-negative bacteria; (O) Staphylococcus homis; (P) Neisseria meningitidis; (Q) Klebsiella pneumonia and Enterobacter cloacae; (R) Enterococcus faecium and Escherichia coli.
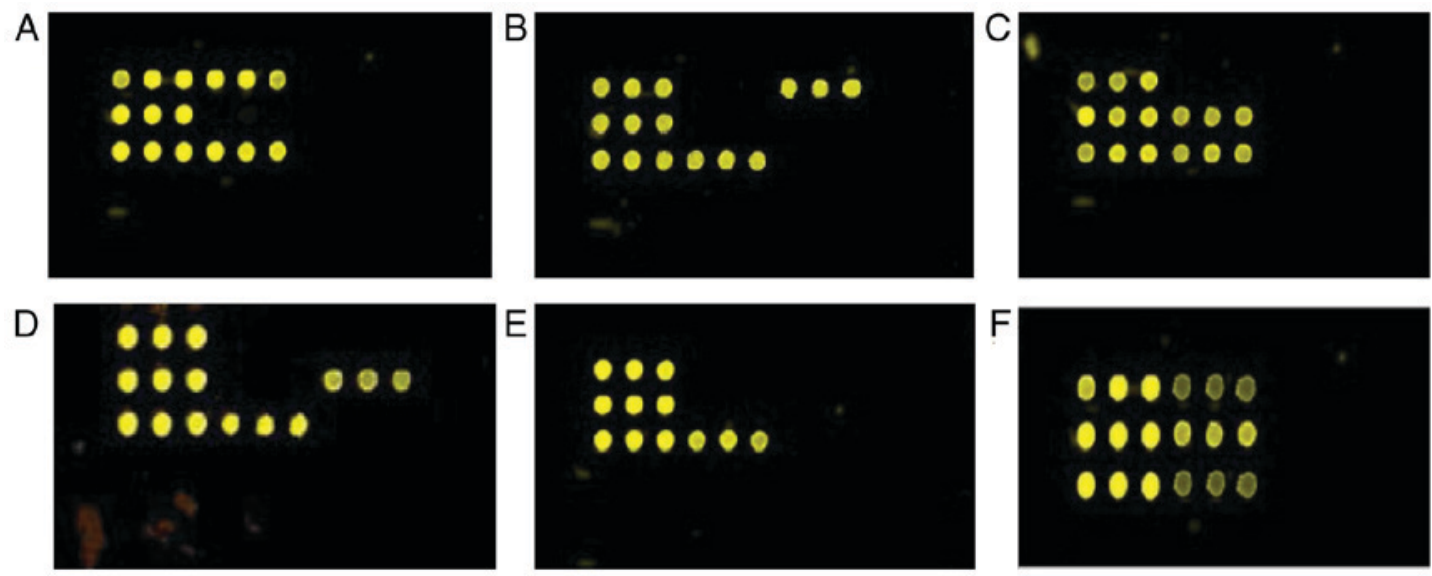

Figure 4. The identification results of fungal species using DNA microarray. (A) Candida albicans; (B) Candida tropicalis; (C) Candida glabrata; (D) Cryptococcus neoformans; (E) Candida parapsilosis; (F) Candida albicans and Candida glabrata.

analysis. Culture testing took 4-7 days to complete the identification, whereas the microarray analysis took only 1 day. Culture testing presented 28 positive results (23 bacteria and 5 fungi), whereas the microarray analysis presented 41 positive cases of bacteria and fungi ( 35 bacteria and 6 fungi). The sensitivity of gene chip was higher than that of the culture method (100 vs. $68.3 \%, \chi^{2}=4.03, \mathrm{P}<0.05$; Table III). The results demonstrated that the specificity, false-positive rate and false-negative rate of microarray technique and culture method were 97.1 vs. $100 \%$, 2.9 vs. $0 \%$, and 0 vs. $31.7 \%$, respectively.

A total of 13 extra pathogens that were determined negative using the culture method, were detected using the gene chip technique, including $P$. aeruginosa $(\mathrm{n}=2)$, E. coli $(\mathrm{n}=2)$, Ochrobactrum anthropi $(\mathrm{n}=2)$, Streptococcus pneumonia $(\mathrm{n}=1)$, A. baumannii $(\mathrm{n}=1)$, Cryptococcus neoformans $(\mathrm{n}=1)$, $16 \mathrm{~S}$ positive $(\mathrm{n}=4)$. The $216 \mathrm{~S}$ positive but unidentified strains were successfully sequenced and confirmed to be Brucella spp., but sequencing of the 2 other strains failed. Of the 13 cases, 2 patients were discharged from hospital prior to etiological diagnosis, 8 patients were treated accordingly based on DNA microarray analysis and recovered, but the 3 more serious cases infected with Ochrobactrum anthropi, P. aeruginosa and A. baumannii succumbed to encephalocele.

\section{Discussion}

The rapid identification of bacteria and fungi can be achieved by the detection of characteristic bacterial genes and fungal genes in CSF specimens, which is important for clinical diagnosis and therapy. Certain non-culture methods have been developed to detect pathogens from CSF samples and may be more sensitive than conventional methods. Previous studies (6,13-16) have usually chosen the 16S rRNA and ITS as a target for universal primers to amplify bacteria and fungi, respectively. The $16 \mathrm{~S}$ rRNA is composed of conserved regions and variable regions, which are widely used in classification and identification of bacteria (14-16). The conserved region and variable region provide the premise for the design of bacterial species-specific primers and probes. In addition, detection of variations of ITS within fungal rDNA spacer regions has been demonstrated to be effective for the identification and classification of fungi (19). The microarray-based virus detection provides a diagnostic tool for viral CNS infections (3). As such, the two technologies of universal primers PCR and DNA microarray were combined in the present study to detect 14 types of bacteria and 4 fungi. The detection was performed in 1 day, facilitating the rapid detection of pathogens causing CNS infection. 
Table III. Positive rates of pathogen detected with DNA microarray and culture method in cerebrospinal fluid.

\begin{tabular}{|c|c|c|c|c|c|c|c|c|c|}
\hline \multirow[b]{3}{*}{ Specimen group } & \multicolumn{4}{|c|}{ DNA microarray } & \multicolumn{4}{|c|}{ Culture } & \multirow[b]{3}{*}{ P-value } \\
\hline & \multicolumn{2}{|c|}{ Positive } & \multicolumn{2}{|c|}{ Negative } & \multicolumn{2}{|c|}{ Positive } & \multicolumn{2}{|c|}{ Negative } & \\
\hline & Bacteria & Fungi & Bacteria & Fungi & Bacteria & Fungi & Bacteria & Fungi & \\
\hline Study group $(n=88)$ & 35 & 6 & 33 & 14 & 23 & 5 & 45 & 15 & $<0.05$ \\
\hline Control group $(n=20)$ & 0 & 0 & 10 & 10 & 0 & 0 & 10 & 10 & $>0.05$ \\
\hline Total $(n=108)$ & 35 & 6 & 43 & 24 & 23 & 5 & 55 & 25 & \\
\hline
\end{tabular}

DNA sequencing was performed for each sample.

The diagnostic probes designed in the microarray covered $>95 \%$ of the bacterial and fungal pathogens responsible for intracranial infection. The DNA microarray exhibited positive fluorescence at the corresponding sites when validating the array with standard strains, except for certain subspecies of coagulase negative Staphylococcus, which exhibited negative signals, possibly because a single probe of coagulase negative Staphylococcus cannot completely detect all subspecies. In our future study, more probes will be designed to detect these subspecies.

The minimum concentration of detection of chips, serving a key role in positive rate, was analyzed in the present study. The results demonstrated that the gene chips were able to identify pathogens at a concentration of $10 \mathrm{cfu} \mathrm{m}^{-1}(10 \mu \mathrm{l} /$ well) for bacteria and $100 \mathrm{cfu} \mathrm{ml}^{-1}(10 \mu \mathrm{l} /$ well $)$ for fungi. In contrast, PCR sequencing was only able to identify pathogens accurately at $>10,000 \mathrm{cfu} \mathrm{ml}^{-1}(10 \mu \mathrm{l} /$ well $)$. Hence, DNA microarray is more sensitive in the detection of all bacteria and fungi, and even more sensitive for detection of Gram-negative bacteria than PCR sequencing. A possible explanation is that Gram-positive bacteria and fungi cell membranes are notoriously difficult to break $(13,19)$, which may lead to poor DNA extraction. The results are notable as the low detection limit will allow direct detection of the DNA from patient specimens, thus improving the detection rate. Furthermore, the results demonstrated that the hybridization signal was linearly dependent on the concentration of targeted microbes. This potentially makes it possible for quantification and automation in the future. The results demonstrated that every probe was specific for its corresponding pathogen, and there was no cross-reaction between them, indicating high specificity in the microarray technique. The results of reproducibility demonstrated that the repeatability of detection using chips had a high CV (5.0-8.0\%). However, the reproducibility was tested with purified DNA and simulated specimens instead of real clinical samples in the present study, therefore the latter may have issues of incomplete DNA isolation, inhibitors being present, etc. which may affect sensitivity.

The rate of false-positive results of microarray technique was $2.9 \%$, which may be due to the contamination of samples. Effective methods for the removal of these substances will be developed and the present protocol will be modified with rigorous use of controls, including the extraction protocol, PCR amplification, hybridization steps and analysis of fluorescence signals.

A total of 23 cases of bacterial and 5 cases of fungal positive culture specimens were identified to have the same results using the microarray assay. A further 12 bacterial and 1 fungal cases of culture-negative specimen were identified, 9 were identified using gene chip hybridization technique, demonstrating the presence of infection. This suggests that microarray technology may be more sensitive than conventional CSF culture methods, which is consistent with other findings $(3,6)$. The 4 unidentified strains that were demonstrated to be positive only in the universal probe sites (16S), which may be due to the pathogens not belonging to any of the species included in the study design. The $216 \mathrm{~S}$ positive unidentified strains were successfully sequenced and confirmed to be Brucella spp., and the infection was controlled with accordingly antimicrobial agents and recovered. Of the 13 cases, 10 patients were recovering and discharged, indicating a higher clinical diagnostic value. The availability of species identification by use of this rapid and sensitive method will enable physicians to treat with appropriate antimicrobial agents in the absence of positive-culture in the CSF in a timely manner, and in turn reduce the inappropriate use of antibiotics and improve patient management.

Based on these findings, the DNA microarray assay of the present study is suitable for detecting and analyzing a large number of pathogens simultaneously, which may facilitate detection and identification of the pathogens responsible for serious infection, especially in the initial screening of patients with suspected CNS infections. Although DNA microarray is not currently the widely accepted clinical diagnostic method, previous studies $(12,20,22)$ and the present study have demonstrated the potential clinical application value of DNA microarray due to its high sensitivity, specificity and reproducibility, while also being less time-consuming. The DNA microarray can also be proposed as a valuable supplement or substitute for the traditional methods (microscopy and culture) in clinical diagnosis of intracranial infections. Furthermore, this method can simultaneously detect a large number of pathogens, suggesting that this technique is helpful in the initial screening of patients with suspected CNS infections. However, the present study was limited by the single-center design and the small number of patients. To improve the clinical value and application of DNA microarray for CNS infections, multi-center large-sample studies with standardized diagnostic criteria and procedures will be conducted in the future. Studies are currently under way to increase the range of species, add $\geq 2$ more probes for each species that can be identified and to apply the small chip directly to CSF specimens and other clinical specimens. 
The findings of the present study suggest that high-density probe of the assay can greatly enhance detection range of species and reduce the cost of testing. Furthermore, it is vital to conduct comprehensive analysis in the future to avoid false-positive results caused by contamination and false-negative results caused by improper DNA extraction methods.

\section{Acknowledgements}

The authors wish to thank their department and research team for their help and dedication.

\section{Funding}

The present study was supported by Beijing Council of Science and Technology (grant no. Z141107002514012).

\section{Availability of data and materials}

The datasets used and/or analyzed during the current study are available from the corresponding author on reasonable request.

\section{Authors' contributions}

$\mathrm{JCa}$ and $\mathrm{PW}$ designed the present study and drafted the manuscript; JCa, SG, JCh and BZ performed the experiments and collected the data; JCa and BZ performed the statistical analysis; JCh collected important background information. RM collected important information regarding CSF pathogens, analyzed and interpreted the data, and made substantial contributions in the design and preparation of the gene chips. The final version of the manuscript has been read and approved by all authors, and each author believes that the manuscript represents honest work.

\section{Ethics approval and consent to participate}

The present study was approved by the Ethics Committee of Xuanwu Hospital, Capital Medical University (Beijing, China). All patients provided written informed consent.

\section{Patient consent for publication}

All patients provided written informed consent for the publication of their data.

\section{Competing interests}

The authors declare that they have no competing interests.

\section{References}

1. Amin M, Ghaderpanah $\mathrm{M}$ and Navidifar T: Detection of Haemophilus influenzae type b, Streptococcus agalactiae, Streptococcus pneumoniae and Neisseria meningitidis in CSF specimens of children suspicious of Meningitis in Ahvaz, Iran. Kaohsiung J Med Sci 32: 501-506, 2016.

2. Taj A and Jamil N: Detection of meningococcal meningitis in cerebrospinal fluid of patients with neurological disorders in government hospitals of Karachi. J Pak Med Assoc 66: 1418-1421, 2016.

3. Bøving MK, Pedersen LN and Møller JK: Eight-Plex PCR and liquid-array detection of bacterial and viral pathogens in cerebrospinal fluid from patients with suspected meningitis. J Clin Microbiol 47: 908-913, 2009.
4. Gade L, Scheel CM,Pham CD,Lindsley MD, Iqbal N, Cleveland AA, Whitney AM, Lockhart SR, Brandt ME and Litvintseva AP: Detection of fungal DNA in human body fluids and tissues during a multistate outbreak of fungal meningitis and other infections. Eukaryot Cell 12: 677-683, 2013.

5. Khater WS and Elabd SH: Identification of common bacterial pathogens causing meningitis in culture-negative cerebrospinal fluid samples using real-time polymerase chain reaction. Int J Microbiol 2016: 4197187, 2016.

6. Sarookhani MR, Ayazi P, Alizadeh S, Foroughi F, Sahmani A and Adineh M: Comparison of 16S rDNA-PCR amplification and culture of cerebrospinal fluid for diagnosis of bacterial meningitis. Iran J Pediatr 20: 471-475, 2010.

7. Xiao X, Zhang Y, Zhang L, Kang P and Ji N: The diagnostic value of cerebrospinal fluid lactate for post-neurosurgical bacterial meningitis: A meta-analysis. BMC Infect Dis 16: 483, 2016.

8. Shen J, Guan Y, Zhang J, Tang J, Lu X and Zhang C: Application of microarray technology for the detection of intracranial bacterial infection. Exp Ther Med 7: 496-500, 2014.

9. Lin AL and Safdieh JE: The evaluation and management of bacterial meningitis: Current practice and emerging developments. Neurologist 16: 143-151, 2010.

10. Rajesh NT, Dutta S, Prasad R and Narang A: Effect of delay in analysis on neonatal cerebrospinal fluid parameters. Arch Dis Child Fetal Neonatal Ed 95: F25-F29, 2010.

11. de Filippis I, de Andrade CF, Caldeira N, de Azevedo AC and de Almeida AE: Comparison of PCR-based methods for the simultaneous detection of Neisseria meningitidis, Haemophilus influenzae and Streptococcus pneumoniae in clinical samples. Braz J Infect Dis 20: 335-341, 2016.

12. Leveque N, Van Haecke A, Renois F, Boutolleau D, Talmud D and Andreoletti L: Rapid virological diagnosis of central nervous system infections by use of a multiplex reverse transcription-PCR DNA microarray. J Clin Microbiol 49: 3874-3879, 2011.

13. Esparcia O, Montemayor M, Ginovart G, Pomar V, Soriano G, Pericas R, Gurgui M, Sulleiro E, Prats G, Navarro F and Coll P: Diagnostic accuracy of a 16 S ribosomal DNA gene-based molecular technique (RT-PCR, microarray and sequencing) for bacterial meningitis, early-onset neonatal sepsis and spontaneous bacterial peritonitis. Diagn Microbiol Infect Dis 69: 153-160, 2011.

14. Srinivasan L, Pisapia JM, Shah SS, Halpern CH and Harris MC: Can broad-range $16 \mathrm{~S}$ ribosomal ribonucleic acid gene polymerase chain reactions improve the diagnosis of bacterial meningitis? A systematic review and meta-analysis. Ann Emerg Med 60: 609-620. e2, 2012.

15. Klindworth A, Pruesse E, Schweer T, Peplies J, Quast C, Horn M and Glöckner FO: Evaluation of general 16S ribosomal RNA gene PCR primers for classical and next-generation sequencing-based diversity studies. Nucleic Acids Res 41: e1, 2013.

16. Liu A, Wang, C1 Liang Z, Zhou ZW, Wang L, Ma Q, Wang G, Zhou SF and Wang Z: High-throughput sequencing of 16S rDNA amplicons characterizes bacterial composition in cerebrospinal fluid samples from patients with purulent meningitis. Drug Des Devel Ther 9: 4417-4429, 2015.

17. Zhong D, Koepfli C, Cui L and Yan G: Molecular approaches to determine the multiplicity of Plasmodium infections. Malar J 17: $172,2018$.

18. Bumgarner R: Overview of DNA microarrays: Types, applications and their future. Curr Protoc Mol Biol 101: 22.1.1-22.1.11, 2013.

19. McCarthy MW and Walsh TJ: PCR methodology and applications for the detection of human fungal pathogens. Expert Rev Mol Diagn 16: 1025-1036, 2016.

20. Rupp S: Microarray technologies in fungal diagnostics. Methods Mol Biol 1508: 385-409, 2017.

21. Ben RJ, Kung S, Chang FY, Lu JJ, Feng NH and Hsieh YD: Rapid diagnosis of bacterial meningitis using a microarray. J Formos Med Assoc 107: 448-453, 2008.

22. Patel A and Cheung SW: Application of DNA microarray to clinical diagnostics. Methods Mol Biol 1368: 111-132, 2016.

23. Raich T and Powell S: Identification of bacterial and fungal pathogens from positive blood culture bottles: A microarray-based approach. Methods Mol Biol 1237: 73-90, 2015.

24. van Veen SQ, Claas EC and Kuijper EJ: High-throughput identification of bacteria and yeast by matrix-assisted laser desorption ionization-time of flight mass spectrometry in conventional medical microbiology laboratories. J Clin Microbiol 48: 900-907, 2010.

This work is licensed under a Creative Commons Attribution-NonCommercial-NoDerivatives 4.0 International (CC BY-NC-ND 4.0) License. 